\title{
Silicon Photonic Sensors incorporated in a Digital Microfluidic System
}

Cristina Lerma Arce*, Daan Witters*, Robert Puers, Jeroen Lammertyn, Peter Bienstman

Cristina Lerma Arce

Ghent University, Dept. of Information Technology

Sint-Pietersnieuwstraat 41, B-9000 Gent, Belgium

Tel: +32 92643318 ,

Web: http://photonics.intec.UGent.be

Email: Cristina.Lermaarce@intec.ugent.be

Daan Witters

KU Leuven

Department of Biosystems

MeBioS Biosensor group

Willem De Croylaan 42, Box 2428

3001 Leuven, Belgium

Tel: +3216321615

Web: http://www.biosensors.be

Email: Daan.Witters@biw.kuleuven.be

Robert Puers

KU Leuven

MICAS-ESAT

Kasteelpark Arenberg 10

3001 Leuven, Belgium

Tel: +32 16-321082

Email: Robert.Puers@esat.kuleuven.ac.be

Jeroen Lammertyn

KU Leuven

Department Biosystems

MeBioS Biosensor group

Willem De Croylaan 42, Box 2428

3001 Leuven, Belgium

Tel: +32 16321459 or +3216321614

Web: http://www.biosensors.be

Email: Jeroen.Lammertyn@biw.kuleuven.be

Peter Bienstman

Ghent University, Dept. of Information Technology

Sint-Pietersnieuwstraat 41, B-9000 Gent, Belgium

Tel: +3292643446

Web: http://photonics.intec.UGent.be

Email: Peter.Bienstman@UGent.be

*Equally contributed to this paper 


\begin{abstract}
Label-free biosensing with silicon nanophotonic microring resonator sensors has proven to be an excellent sensing technique for achieving high-throughput and high sensitivity, comparing favorably with other labeled and label-free sensing techniques. However, as in any biosensing platform, silicon nanophotonic microring resonator sensors require a fluidic component which allows the continuous delivery of the sample to the sensor surface. This component is typically based on microchannels in PDMS or other materials which add cost and complexity to the system.

The use of microdroplets in a digital microfluidic system, instead of continuous flows, is one of the recent trends in the field, where micro- to picoliter-sized droplets are generated, transported, mixed and split, thereby creating miniaturized reaction chambers which can be controlled individually in time and space. This avoids cross-talk between samples or reagents and allows fluid plugs to be manipulated on reconfigurable paths, which cannot be achieved using the more established and more complex technology of microfluidic channels where droplets are controlled in series. It has great potential for high-throughput liquid handling, while avoiding on-chip cross contamination.

We present the integration of two miniaturized technologies: label-free silicon nanophotonic microring resonator sensors and digital microfluidics, providing an alternative to the typical microfluidic system based on microchannels. The performance of this combined system is demonstrated by performing proof-of-principle measurements of glucose, sodium chloride and ethanol concentrations. These results show that multiplexed real-time detection and analysis, great flexibility, and portability make the combination of these technologies an ideal platform for easy and fast use in any laboratory.
\end{abstract}

\title{
Keywords
}

Silicon nanophotonics ring resonators sensors, Silicon-on-insulator (SOI), Digital Microfluidics, Electrowetting-onDielectric (EWOD), Microdroplets.

\section{Introduction}

Monitoring of molecular interactions provides critical information for medical diagnosis, drug development and environmental protection. The presence and concentration of target molecules, as well as receptor-target binding affinity and kinetics, can be measured by monitoring the binding between molecules of interest, e.g., proteins, biomarkers, and complementary DNA strands. Because of the complex nature of most biological interactions, sensor arrays capable of multiplexed measurements are often required. Miniaturization, low cost and portability are desirable attributes for such sensing systems [1].

Silicon-on-insulator (SOI) is a material system with many assets for these applications. First of all, it has a high refractive index contrast permitting very compact sensors of which many can be incorporated on a single chip, enabling multiplexed sensing. Secondly, silicon-on-insulator photonic chips can be made using CMOS-compatible process steps, allowing for a strong reduction of the chip cost by high volume fabrication [2]. These sensor chips can therefore be disposable, meaning that the chip is only used once, avoiding complex cleaning of the sensor surface after use, which is required to avoid cross contamination when used for medical diagnostics.

A crucial component in most of these photonic biosensors is the transducer that can transform a refractive index change in its environment to a measurable change in its optical transmission. Microring resonator sensors stand out as prime candidates for such transducers among other technologies such as Fabry-Perot cavities, microdisks, or photonic crystal cavities, for achieving high performance in a robust manufacturable manner [3]. These label-free evanescent field sensors have seen rapid development in recent years [3-6]. 
However, not only the transducer but also the microfluidic system is an essential element for such sensing systems. The use of microfluidic channels for delivery of the sample in continuous flow to the sensor surface is one of the most critical steps in the integration of biosensors and microfluidics for point-of-care applications. This integration is not straightforward, since the interface with the outside world needs special attention, and care needs to be taken to avoid leaks and channel clogging.

Digital microfluidics is an emerging technology similar to the more established technology of microfluidic channels in that both can perform lab analyses with much smaller sample volumes than in bench-scale methods. Both methods are capable of manipulating individual microliter- to nanoliter-sized droplets [7]. Although different droplet-actuation mechanisms have been developed, electrowetting-on-dielectric (EWOD) is unique because all elementary fluidic operations (droplet dispensing, splitting, merging, transport, and mixing) are performed on reconfigurable paths of buried actuation electrodes with high flexibility without the need of moving parts such as pumps or valves. EWOD is based on modulating of the interfacial tension between a liquid and an electrode buried underneath a hydrophobic dielectric layer. By establishing an electric field in the dielectric layer on one side of the droplet, the contact angle is locally changed from hydrophobic to hydrophilic, causing the droplet to move [8-10].

In microfluidic channels droplets are controlled in series, while in digital microfluidics they are addressed individually, thereby avoiding cross-talk between samples or reagents which provides a very efficient way to prevent cross contamination. The most popular applications of EWOD-based digital microfluidic chips are bioassays such as DNA extraction and amplification [11], cell-based assays [12-13], enzymatic assays [14-17] and immunoassays [18].

Few research results have been reported on the integration of optical sensing systems in digital microfluidic chips $[19,20]$. The first integration of microring sensors on digital microfluidic chips has already been demonstrated [21, 22], although these systems rely on very complex fabrication procedures for making integrated optical detectors and bulky optical fiber connections to couple light into the system.

Here, we demonstrate how digital microfluidics can be used for effective fluid delivery to nanophotonic microring resonator sensors fully constructed in SOI. We show how SOI chips containing the microring resonator sensors can be modified to directly serve as the top plate of double-plate digital microfluidic chips without affecting microfluidic chip performance, while enabling integrated optical sensing of molecules near the chip surface. Moreover, by using grating couplers to couple light into and out of the SOI chips, they can be used in a plug-and-play fashion without the need for external connections. Combination of both technologies offers unique advantages and shows great potential for performing label-free biosensing, as cross-contamination, microchannel clogging and air bubble formation are avoided, while minimal sample waste is generated. Moreover, since digital microfluidics is inherently an array-based technique and photonic ring resonator sensors are commonly used in arrays as well, the proposed combined system is ideal for array-based biochemical applications.

\section{Materials and Methods}

\subsection{Digital microfluidic system}

The cross-section of the EWOD lab-on-a-chip is shown in Figure 1. Many EWOD lab-on-a-chip device configurations exist, mainly differing in the actuation electrode architecture and the type of dielectric material used. This dielectric layer is a crucial element in the EWOD lab-on-a-chip configuration, as it isolates the liquid droplet from the actuation electrodes, thereby preventing electrolysis when a voltage is applied across the droplet [23, 24]. Below, we describe the fabrication process for our EWOD lab-on-a-chips based on the protocol of [25]. 

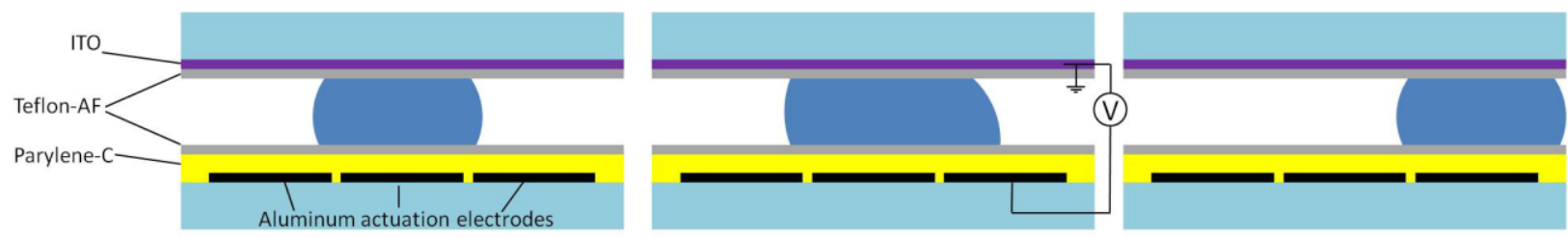

Figure 1. A diagram of the cross-section of an EWOD lab-on-a-chip and the electrowetting principle. When a voltage is applied to an actuation electrode, the droplet is attracted toward this activated region due to the generated imbalance in interfacial tension near the surface above the actuated electrode [16].

The digital microfluidic platform was produced by means of standard photolithographic techniques. For the bottom part of the chip, a 100-nm thick aluminum layer was sputtered on a glass wafer and subsequently patterned using standard photolithographic techniques. All electrodes were $1.4 \times 1.4 \mathrm{~mm}^{2}$. The aluminum layer was covered by a 2.8 $\mu \mathrm{m}$ dielectric Parylene-C layer deposited using chemical vapor deposition. This insulating layer was rendered hydrophobic by spin-coating a layer of Teflon-AF (300 nm thickness) on top. The top part of the digital lab-on-achip consisted of a glass plate coated with a $120 \mathrm{~nm}$ layer of transparent indium tin oxide (ITO) which was made hydrophobic with a Teflon-AF layer of approximately $300 \mathrm{~nm}$. Standard tape with a thickness of $80 \mu \mathrm{m}(3 \mathrm{M}$, St. Paul, MN, USA) was used as spacer between top and bottom plates. Figure 2 shows this digital microfluidic platform.
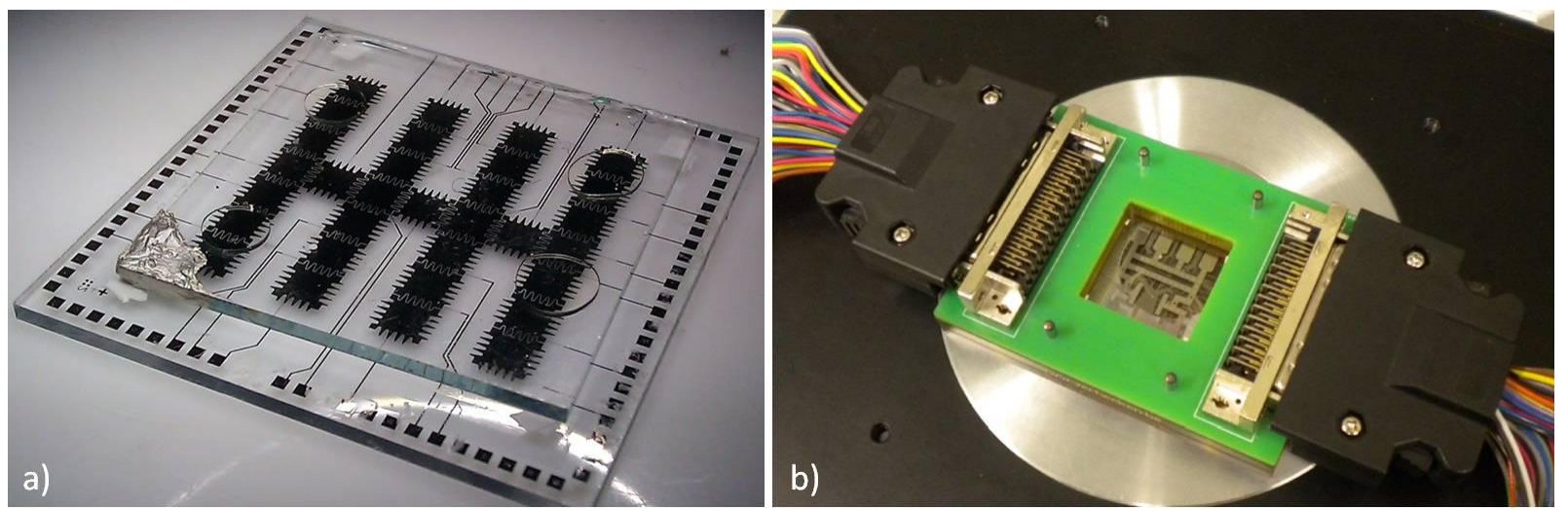

Figure 2. a). Photograph of the digital microfluidic chip with a standard ITO top plate on top, visualizing the sandwiched droplets. b). Assembled EWOD platform demonstrating the chip holder with electrical connections.

Droplets were sandwiched between the two plates. To activate the electrowetting-on-dielectric mechanism, a 130V DC actuation voltage was used, while the electrode actuation sequence, activation time and relaxation time were controlled by means of homemade programs in MATLAB (The MathWorks, Inc., Natick, MA, USA) and LabView (National Instruments Corp., Austin, TX, USA). When a voltage is applied to the system, a surface tension gradient at the droplet surface is evoked which attracts the droplet towards the activated region. As such, the droplet movement can be freely controlled along a pattern of electrodes as shown in Online Resource 1.

2.2. Nanophotonic ring resonator sensors, chip layout and setup.

The photonic chip was fabricated in silicon-on-insulator with $2 \mu \mathrm{m}$ buried oxide and a 220-nm silicon top layer with CMOS-compatible 193-nm optical lithography and dry etching [2]. The resonators consist of 450-nm-wide singlemode waveguides, with $5 \mu \mathrm{m}$ bend radius, $2 \mu \mathrm{m}$ long directional couplers and a gap of $180 \mathrm{~nm}$ between the waveguides. 
The layout of the chip is illustrated in Figure 3 [4]. Four rings are connected to one common input waveguide, each of them having a dedicated drop signal port. Three of these four ring series are placed independently next to the other. The three input waveguides are simultaneously addressed through vertical grating couplers [26] with a 2 mmwide collimated beam from a tunable laser source. The output signals of the ring resonators are near-vertically coupled to free space by means of integrated grating couplers and are imaged with an infrared camera.
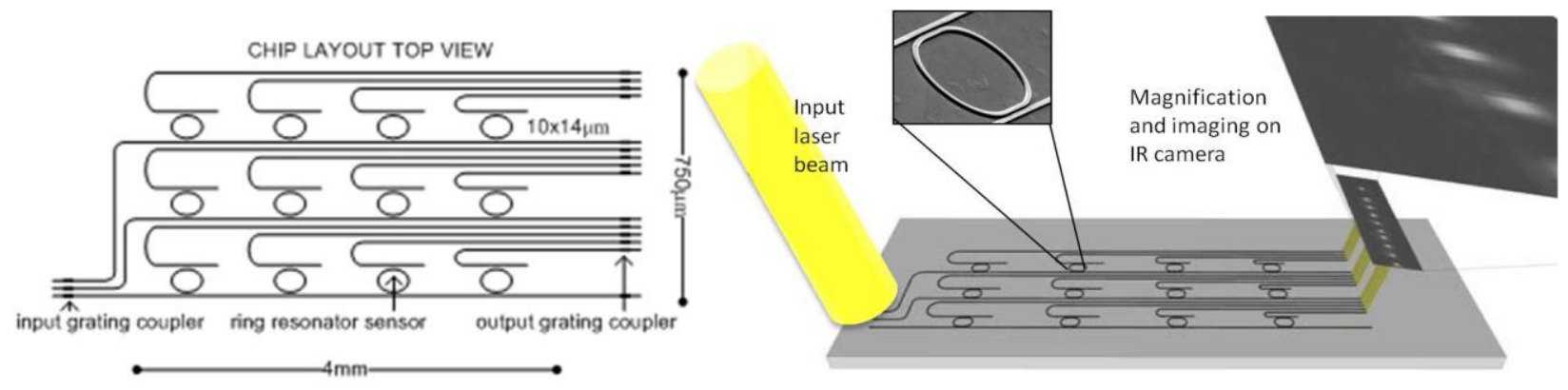

Figure 3 Left. Chip layout top view. Four rings are connected to one common input waveguide, each of them having a dedicated drop signal port. Three of these four ring series are placed independently next to the other. Right: Setup: A SANTEC TSL-510 tunable laser was used as a light source. The three input waveguides are simultaneously addressed through vertical grating couplers with a $2 \mathrm{~mm}$-wide collimated beam from a tunable laser source. The output signals of the ring resonators are nearvertically coupled to free space by means of integrated grating couplers and are imaged with an infrared camera. [4]

The shallow-etched gratings are part of the chip design and have a maximum coupling efficiency of $31 \%$ at $1.55 \mu \mathrm{m}$ wavelength (40-nm 1-dB bandwidth) for $10^{\circ}$ off-vertical coupling angle. Because the bandwidth of the grating couplers is larger than the free spectral range of the resonators, the grating couplers do not limit the number of resonators placed in series. This optical setup allows for very high alignment tolerances, measures the spectrum of all the ring resonators in parallel and therefore forms no limitation for high-throughput sensing.

A SANTEC TSL-510 tunable laser (Santec Corporation, Aichi, Japan) was used as a light source. The transmitted light was detected by a XenICs infrared camera (Xenics, Leuven, Belgium). The input power was chosen so that the intensity of the resonance peaks corresponds to the pixel saturation level to obtain a maximum signal-to-noise ratio. We have developed software that captures an image for every wavelength step and stores the maximum intensity values within each dedicated area that overlaps with an output grating coupler spot. Post-processing consists of fitting of the spectra to their theoretical shape and tracking of these resonance peaks over time.

\subsection{Integrating both technologies}

The SOI chip containing the array of ring resonator sensors described above was incorporated into the digital microfluidic system by replacing the top glass plate of the platform. Figure 4 illustrates how the chip was placed upside down, squeezing the liquid droplets against the bottom plate containing the buried electrodes.

In order to guarantee the hydrophobicity of the SOI chip surface, which is crucial for performing droplet manipulations efficiently, and at the same time ensure the contact of the sensors with the droplets, a layer of TeflonAF (300 nm thickness) was coated on the SOI chip and subsequently patterned. Hereto, the Teflon-AF surface was

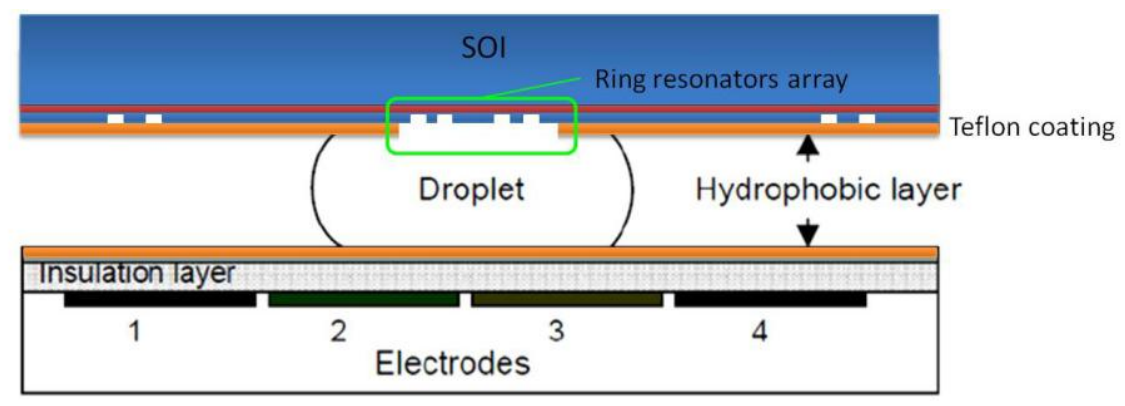

Figure 4: Incorporation of the SOI chip containing an array of microring resonator sensors into the digital microfluidic system by replacing the top glass plate of the platform. 
activated with $\mathrm{O}_{2}$-plasma to allow photoresist to be spincoated on top. This thin layer of photoresist was then patterned by using standard photolithography to expose the regions covering the ring resonators. Next, the TeflonAF covering the ring resonators was locally removed with reactive ion etching using $\mathrm{O}_{2}$-plasma. Some ring resonators were left covered with Teflon-AF in order to provide a reference to compensate for environmental drift.

Figure 5b shows how the SOI chip containing arrays of microring resonator sensors (Figure 5a) was aligned on top of the digital microfluidic chip so that the sensor array coincided with one arm of the electrode pattern underneath in the bottom plate of the microfluidic chip, thereby ensuring contact of the ring resonators with the liquid droplets.
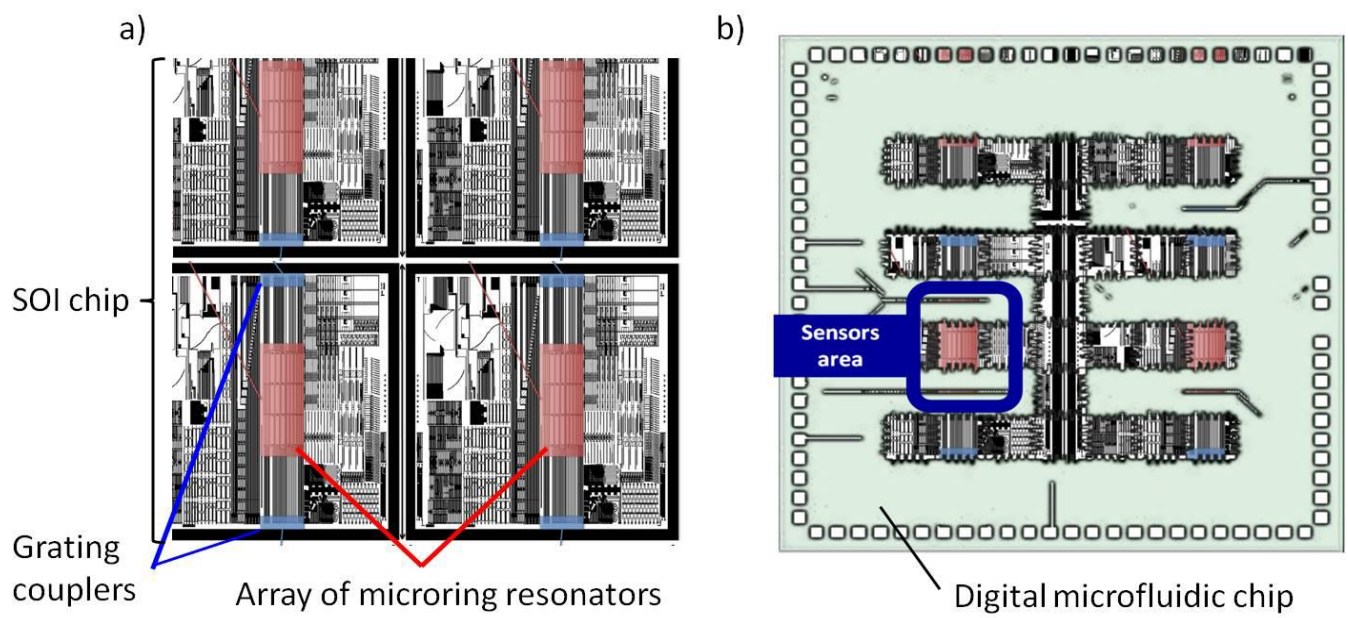

Figure 5 a) SOI chip containing arrays of microring resonator sensors. The relevant microring sensor arrays and grating couplers are indicated in red and blue, respectively. b) Alignment of the SOI chip on the digital microfluidic chip. The SOI chip was aligned so that one of sensor arrays coincided with one arm of the electrodes pattern underneath, thereby ensuring contact of the ring resonators with the liquid droplets.

As described in Section 2.2, grating couplers were used to couple the light from a tunable laser into the chip and couple it out to be detected by an infrared camera. A new aspect with respect to our previous work [4] is that now, since the chip is placed up-side down, light needs to be coupled in and out through the $750-\mu \mathrm{m}$ thick silicon substrate. Silicon is considered practically transparent for the wavelength used $(1.55 \mu \mathrm{m})$.

However, to reduce the scattering of the rough substrate surface and to facilitate the alignment of the laser beam and the detection of the light coupled out from the chip, a few simple processing steps were done in advance: the silicon substrate was thinned down to $300 \mu \mathrm{m}$ by chemical mechanical grinding and afterwards a chemical mechanical polishing step was performed in order to attain a smooth surface. Figure 6 shows an image from the IR camera

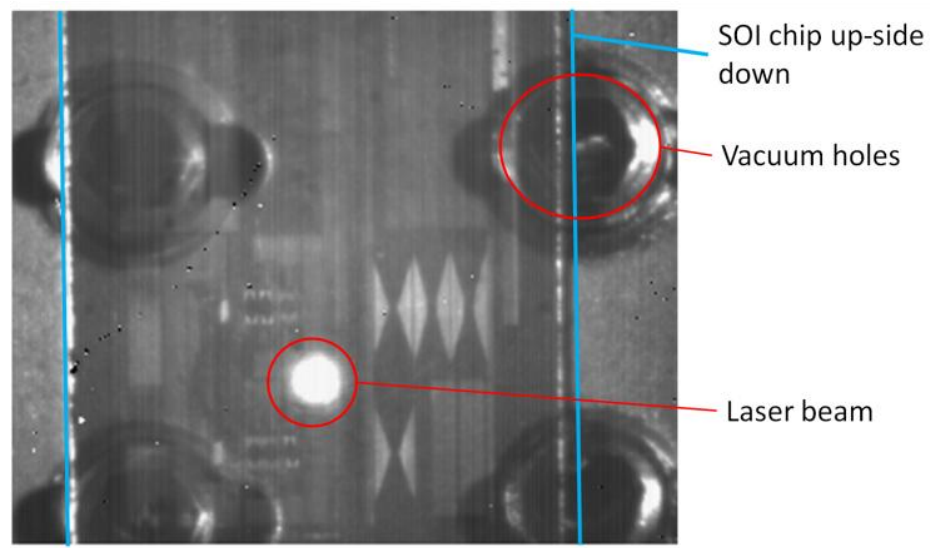

Figure 6. IR camera image. The chip is placed up-side down on a vacuum chuck. The laser beam and the vacuum holes of the chuck at the other side of the chip are clearly visible. The different photonic circuits on the SOI chip are distinguished as shadows and lighted areas in the picture. 
where the chip is placed up-side down on a chuck that uses vacuum to keep the sensor fixed in place. The different photonic circuits of the SOI chip, the laser beam and even the vacuum holes of the chuck at the other side of the chip are clearly visible.

\subsection{Bulk sensing experiments}

As a proof-of-principle to show the capabilities of the combined system, we measured the sensitivity for refractive index changes of aqueous solutions and compared it with previous measurements for the same array of sensors using typical microfluidics based on microchannels [27] and the simulations for bulk refractive index changes done in Fimmwave (Photon Design, Oxford, UK).

To demonstrate the proof-of-principle of on-chip sensing, three different sets of experiments were performed, each with a different chemical compound whose concentration in deionized (DI) water was to be determined. For this purpose, we chose sodium chloride, glucose and ethanol. For each of these compounds, 3 different droplets with different concentrations were prepared (Table 1). For this proof-of-principle experiment, droplets were pipetted manually on the chip surface. However, dilutions of these compounds can also be automated on-chip by executing

\begin{tabular}{|c|c|c|c|c|c|c|c|}
\cline { 2 - 8 } \multicolumn{1}{c|}{} & \multicolumn{2}{c|}{ Sodium chloride } & \multicolumn{2}{c|}{ Glucose } & \multicolumn{2}{c|}{ Ethanol } \\
\cline { 2 - 8 } \multicolumn{1}{c|}{} & Volume & $\begin{array}{c}\text { Mass } \\
\text { concentration } \\
(\%)\end{array}$ & $\begin{array}{c}\text { Refractive } \\
\text { index }\end{array}$ & $\begin{array}{c}\text { Mass } \\
\text { concentration } \\
(\%)\end{array}$ & $\begin{array}{c}\text { Refractive } \\
\text { index }\end{array}$ & $\begin{array}{c}\text { Mass } \\
\text { concentration } \\
(\%)\end{array}$ & $\begin{array}{c}\text { Refractive } \\
\text { index }\end{array}$ \\
\hline Droplet 1 & $5 \mu \mathrm{l}$ & 0 & 1.3105 & 0 & 1.3119 & 0 & 1.3333 \\
\hline Droplet 2 & $5 \mu \mathrm{l}$ & 1.01 & 1.3122 & 0.97 & 1.4559 & 10 & 1.3395 \\
\hline Droplet 3 & $5 \mu \mathrm{l}$ & 1.84 & 1.3137 & 1.97 & 1.6044 & 20 & 1.3469 \\
\hline
\end{tabular}

Table 1. Different concentrations and refractive index of the droplets used in the experiments. For each chemical compound (sodium chloride, glucose, ethanol) 3 droplets of $5 \mu 1$ with different concentrations were prepared. The refractive index of sodium chloride and glucose was derived from [28] and the refractive index of ethanol from [29].

multiple merging and splitting steps [15]. For each droplet, we performed simultaneous measurements with 12 different ring resonators in the array under the droplet. Finally, each of these measurements was repeated 3 times. In total, this represents $3 \times 3 \times 12 \times 3=324$ measurements, from which the sensor sensitivity and sensor variability can be derived.

In each array, four ring resonators where left covered with Teflon-AF, to isolate them from external drifts and to provide a reference for each measurement. The refractive index of sodium chloride and glucose was derived from [28] and the refractive index of ethanol from [29].

The measurements were performed as follows, e.g. for the sodium chloride solutions: a droplet of DI-water was transported on the digital microfluidic platform to the sensors area. Our system started measuring. Subsequently, the droplet of water was moved, leaving a free route for the second droplet with a first sodium chloride concentration. Since the read-out system performs continuous measurements in time, we measured in air during the switching of droplets, causing a big shift in the resonance wavelength, which was discarded. When the second droplet reached the
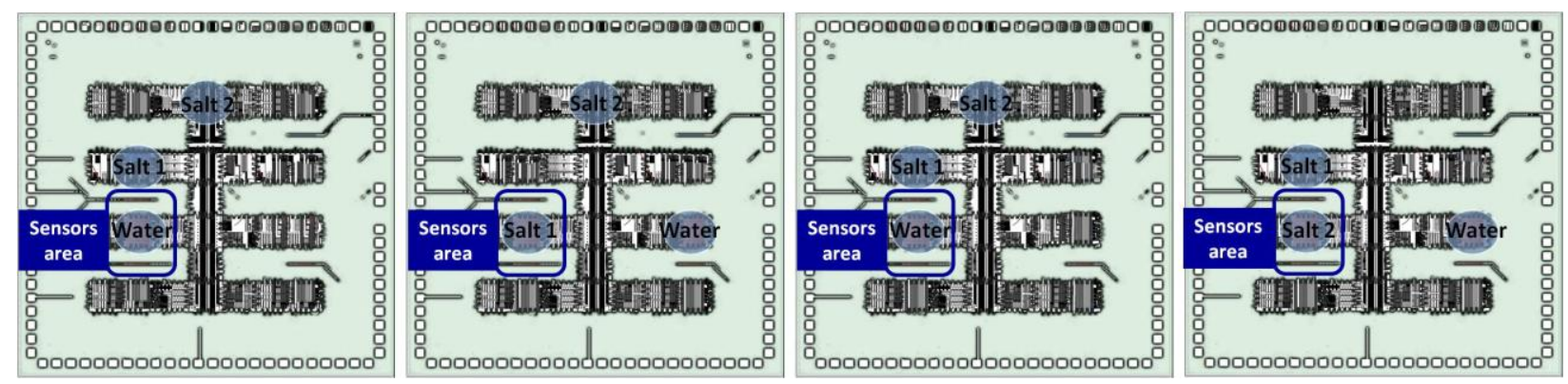

Figure 7. Measurement process: Our system started measuring when a droplet of DI-water was transported in the digital microfluidic platform to the sensors area. Subsequently, that droplet was switched by a droplet with a certain sodium chloride concentration which was measured once it reached the sensing area. This process was repeated by measuring again the water droplet and a third droplet with a different sodium chloride concentration. 
sensing area, the measured signal will shift again to a similar wavelength as measured during the previous droplet, since the refractive index of different watery solutions does not differ to a large extent. This process was repeated by measuring again the water droplet and a third droplet with a different sodium chloride concentration, as illustrated in Figure 7.

Part of this measurement was recorded with the IR camera which is shown in the online video [Online Resource 2].

This same process was repeated for measuring glucose and ethanol concentrations in DI-water in order to confirm the behavior of the system for different refractive index materials and samples, while guaranteeing reproducibility. One photonic chip was used to perform the three repetitions of the measurement of each solution. Thus, three different photonics chips (one per each solution) were used in total to perform the experiments, while the digital microfluidic platform was reused.

\section{Results}

The Teflon-AF pattern on the array of ring resonator sensors is shown in Figure 8. It is a crucial aspect for the experiments performed, since it guarantees the hydrophobicity of the SOI chip surface and at the same time ensures the contact of the sensors with the droplets. In each array of sensors, four ring resonators where left covered with Teflon-AF to isolate them from external drifts. The measured signal of these rings shows a standard deviation smaller than $2 \%$, providing thus an excellent reference for each measurement.

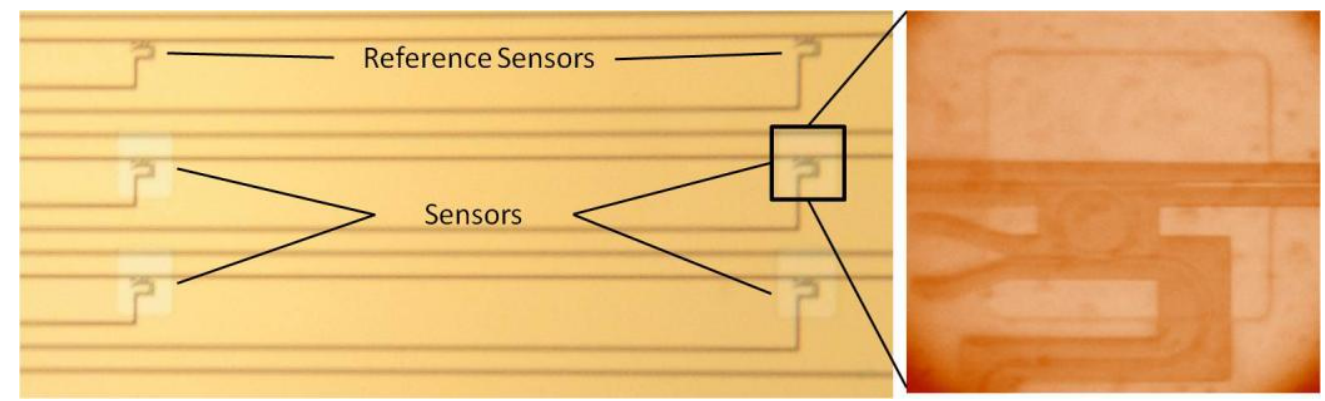

Figure 8. The SOI chip surface was coated with a 125-nm layer of Teflon-AF and consequently patterned in order to guarantee the hydrophobicity of the SOI chip surface and at the same time ensure the contact of the sensors with the droplets. This is a crucial aspect for the measurements since it provides an excellent reference to compensate for environmental drift.

Another important aspect on these measurements is that the SOI is placed up-side down, so light needs to be coupled in and out through the silicon substrate. Figure 9 provides a comparison between two generic measurements from the front side and the backside of the SOI chip. Certain intensity attenuation is observed. However we consider this degradation not relevant for the measurements since the resonances of the rings are clearly measurable.

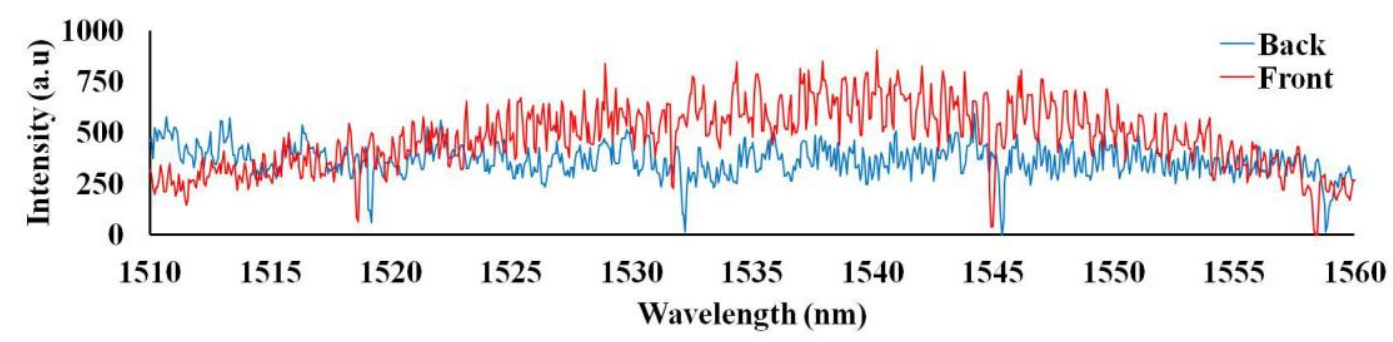

Figure 9. Comparison of the transmission spectra of a ring resonator measured from the front side and backside. The attenuation observed when measuring from the backside of the SOI chip is not relevant for the measurements since the resonances of the ring are still clearly measurable. 
Figure 10 shows the evolution in time of the resonance wavelength shift of the ring resonators during the measurements with different sodium chloride concentrations. Each color corresponds to one sensor. The shift of the resonance wavelength is easily measurable when changing from water to salt solution and vice versa, proving the reproducibility of the experiment. The variability between sensors was determined to be smaller than $8 \%$. During the switch of droplets the measurement had to be done in air (as explained in previous section) which explains the absence of data points in the graph since the change of refractive index from aqueous solutions to air implies big shift in the resonance wavelength.

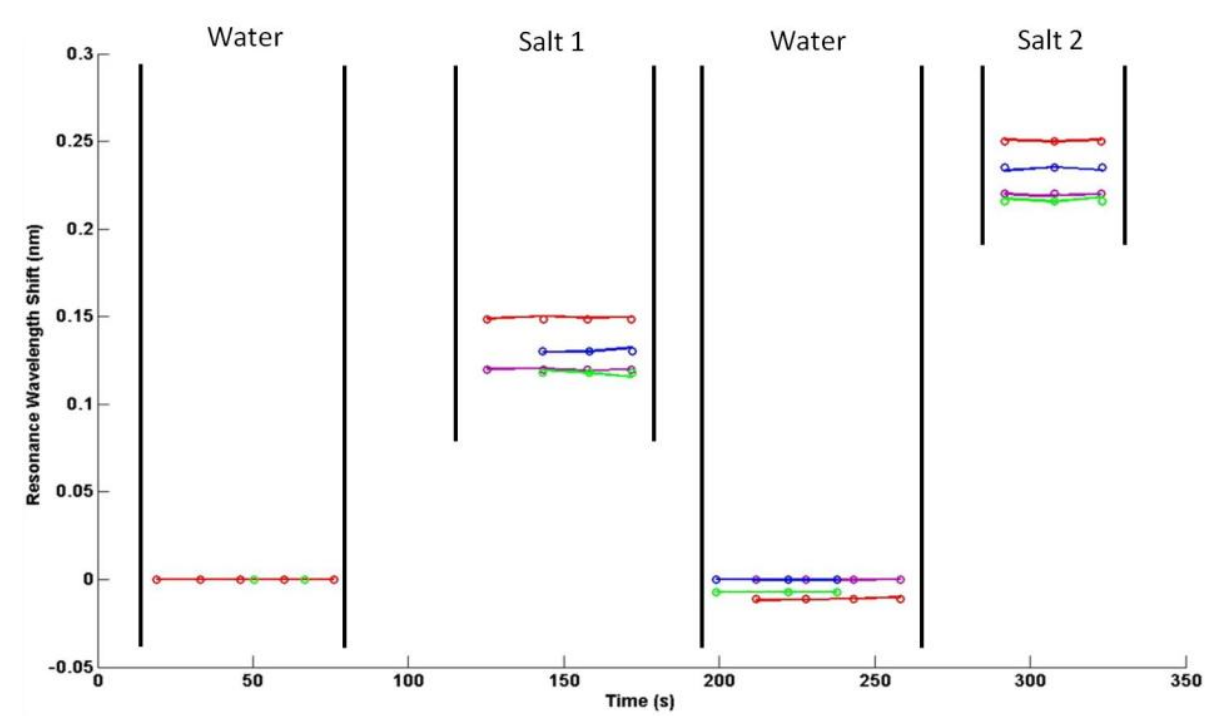

Figure 10. Evolution in time of the resonance wavelength shift of the ring resonators during the measurements of different sodium chloride concentrations. Each color corresponds to one sensor. When changing from water to salt solution and vice versa the shift of the resonance wavelength is easily measurable, proving the reproducibility of the experiment.

Analyzing these shifts as a function of the refractive index unit (RIU) of the droplets, we observed a very close correspondence between the simulations and the measurement results (Figure 11). $78 \mathrm{~nm} / \mathrm{RIU}$ is the sensitivity that these ring resonators show for refractive index change simulations, which was also measured and proved in [27] using the typical microfluidcs system based on microchannels. The measurements performed in this digital microfluidic system show a sensitivity of $77 \pm 0.6 \mathrm{~nm} / \mathrm{RIU}$, which shows that the performance of the transducer does not suffer from being integrated in a digital microfluidics system.
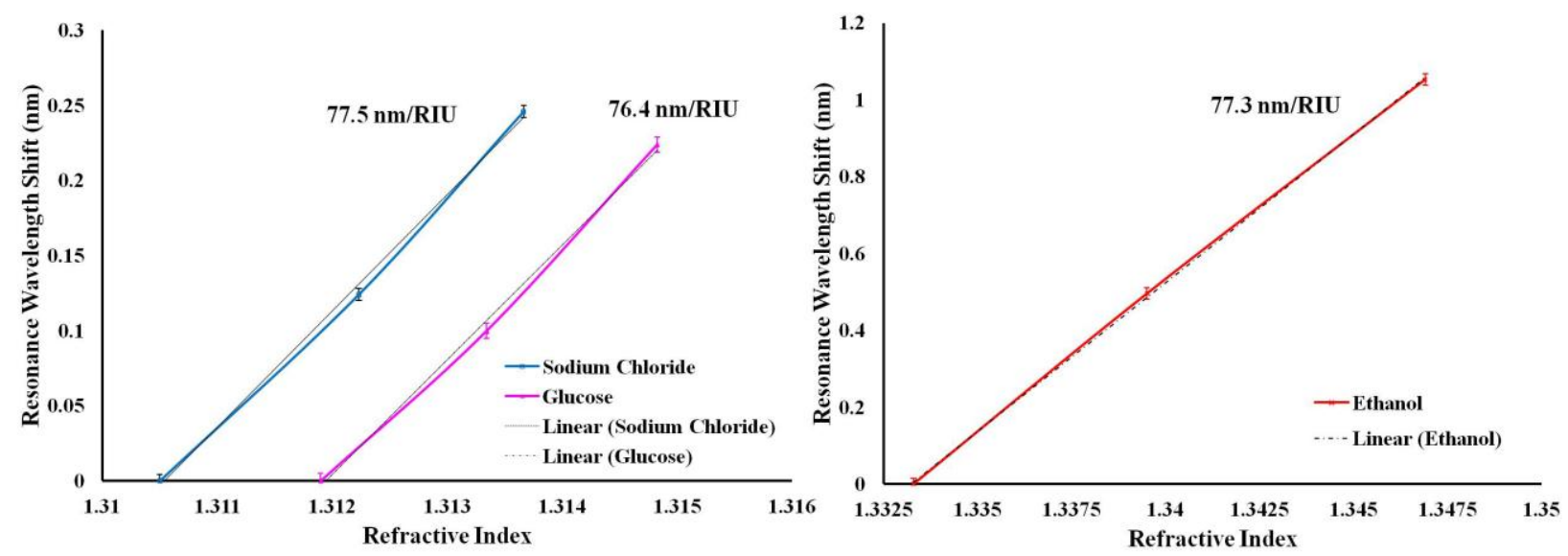

Figure 11. Three experiments with different sodium chloride, glucose and ethanol concentrations were performed. Each line corresponds to one of these experiments. Error bars indicate the standard deviations based on 3 repetitions. Three photonics chips (one per each solution) were used to perform the three experiments. A sensitivity of $77 \pm 0.6 \mathrm{~nm} / \mathrm{RIU}$ is determined taking into account the slope of the linear fitting for the three lines, showing a perfect match with simulations and previous measurements of the same sensors using the typical more complex microfluidic system [27]. 


\section{Conclusions}

We have presented the combination of label free-silicon nanophotonic microring resonator sensors in SOI with digital microfluidics, providing an alternative to the typically costly and complex microfluidic system based on microchannels used for liquid sample delivery to microring resonators.

The alternative presented here provides a cheap and flexible method for the integration of photonic sensors and microfluidics. The presented integration of SOI microring resonators enables plug-and-play ease of use, as no physical connection is needed between the optical chips and the light source and detection unit, while SOI chips can be fabricated in mass production volumes. This enhances disposability of the chips which is beneficial for performing bio-assays where cross contamination is of great concern. We have proved the excellent performance and compatibility of this combined system, showing no degradation of the sensor performance with respect to simulations or previous measurements of the same sensors using the typical, more complex microfluidic system [27]. This combination allows for multiplexed real-time detection and analysis. Its great flexibility, portability and disposability make it ideal for easy and fast use in any laboratory.

Future work will consist of biofunctionalization of the microring sensor surface by using silane chemistry. This will allow us to functionalize the sensor surface with specific capture molecules without affecting the surface hydrophobicity and hence sensor performance. This will open up many possibilities for performing multiplexed antibody detection and bio-assays on the proposed system, and further exploit its potential for performing miniaturized analysis. This can pave the way for a novel integrated platform for diagnostics and life sciences in general, as the platform can be exploited for assessing molecular interactions and cell-based studies in a multiplexed high-throughput context.

\section{Acknowledgements}

Part of this work was supported by the Flemish Fund for Scientific Research (FWO Vlaanderen) under project number 3G099711. The authors would like to acknowledge EFRO Interreg NanosensEU for its financial support, ePIXfab (www.epixfab.eu) for the fabrication of the optical chip and Katrien De Vos and Tom Claes for designing the sensors. 


\section{References}

[1] Xu D.X, Vachon M, Densmore A. (2010) Opt. Lett. 35 (16)

[2] Selvaraja S. K, Jaenen P, Bogaerts W, Van Thourhout D, Dumon P, and Baets R, (2009) J. Lightwave Technol 27: 4076-4083

[3] Iqbal M, Gleeson M.A, Spaugh B, Tybor F, Gunn W. G, Hochberg M, Baehr-Jones T, Bailey R.C, Gunn L. C. (2010) IEEE J. Quant. Electr., 16(3) : 654-661

[4] De Vos K, Girones Molera J, Claes T, De Koninck Y, Popelka S, Schacht E., Baets R, Bienstman P. (2009) IEEE Phot Journal, 1(4): 225-235

[5] Claes T, Bogaerts W, Bienstman P, (2011) Opt. Lett. 36(17): 3320-3322

[6] Xu D.-X, Densmore A, Delâge A, Waldron P, McKinnon R, Janz S, Lapointe J, Lopinski G, Mischki T, Post E, Cheben P, and Schmid J. H, (2008) Opt. Exp. 16: 15137

[7] Wheeler A. R, (2008) Science 322: 539

[8] Berge B, C. R. (1993) Acad. Sci. II 317: 157

[9] Pollack P. M. G, Shendorov A, Fair R. B, (2000) Appl. Phys. Lett 77: 1725

[10] Fair R. B, (2007) Microfluid. Nanofluid. 3: 245

[11] Abdelgawad M, Freire S. L, Yang H., Wheeler A. R., (2008) Lab Chip 8: 672

[12] Witters D, Vergauwe N, Vermeir S, Ceyssens F, Liekens S, Puers R, Lammertyn J, (2011) Lab Chip11: 27902794

[13] Barbulovic-Nad I, Yang H, Park P. S, Wheeler A. R, (2008) Lab Chip 8: 519

[14] Srinivasan V, Pamula V. K, Fair R.B, (2004) Lab Chip 4: 310

[15] Vergauwe N, Witters D, Atalay Y, Verbruggen B, Vermeir S, Ceyssens F, Puers B, Lammertyn J, (2011) Microfluid. Nanofluid. 11: 25

[16] Vergauwe N, Witters D, Ceyssens F, Vermeir S, Verbruggen B, Puers B, Lammertyn J, (2011) J. Micromech. Microeng. 21: 054026

[17] Witters, D, Vergauwe N, Ameloot R, Vermeir S, De Vos D, Puers B, Sels B, Lammertyn J, (2012) Adv. Mat., 24 (10): 1316-1320.

[18] Sista R, Hua Z, Thwar P, Sudarsan A, Srinivasan V, Eckhardt A, Pollack M, Pamula V. K, (2008) Lab Chip 8: 2091

[19] Lin L, Evans R D, Jokerst, N M, Fair, R B, (2008) IEEE Sens. J., 8: 628-635

[20] Malic L, Veres T, Tabrizian M, (2008) Lab Chip 9: 473-475

[21] Luan L, Evans R D, Schwinn D, Fair R B, Jokerst N M, (2008) Proc. IEEE Lasers Electro-Opt. Soc. 21st Ann. Meet., Nov. 2008, 259-260 
[22] Luan L, Royal M W, Evans R, Fair, R B, Jokerst N M, (2011) IEEE Sensors Journal, 12: 1794-1800

[23] Liu H, Dharmatilleke S, Maurya D K and Tay A A O, (2009) Microsyst. Technol. 16: 449-60

[24] Lin Y-Y, Evans R D,Welch E, Hsu B-N,Madison A C and Fair R B, (2010) Sensors Actuators B 150: 465-70

[25] Pollack M G, Shenderov A D and Fair R B, (2002) Lab Chip 2: 96-101

[26] Bogaerts W, Baets R, Dumon P, Wiaux V, Beckx S, Taillaert D, Luyssaert B, Van Campenhout J, Bienstman P, and Van Thourhout D, (2005) J. Lightw. Technol. 23, 1: 401-412

[27] De Vos K, Bartolozzi I, Schacht E, Bienstman P, Baets R, (2007) Opt Exp, 15(12): 7610-7615

[28] Su H, Xu G,H, (2007) Sensors Actuators B 126: 579-582

[29] R. Belda, J. V. Herraez and O.Diez (2005) Physics and Chemistry of Liquids, 43(1): 91-101 